\title{
Analytical energy gradient of high-spin multiplet state calculated by the SAC-CI method
}

\author{
Mayumi Ishida, Kazuo Toyota, Masahiro Ehara, Hiroshi Nakatsuji * \\ Department of Synthetic Chemistry and Biological Chemistry, Graduate School of Engineering, Kyoto University, \\ Sakyou-ku, Kyoto 606-8501, Japan
}

Received 17 September 2001; in final form 18 October 2001

\begin{abstract}
A method of calculating analytical energy gradient of high-spin multiplet state by the SAC-CI (symmetry-adaptedcluster configuration-interaction) method is developed and implemented. This method is expected to be a powerful tool in studying the dynamics and properties of molecules having high spin-multiplicities. Good performance of this method is shown for the quartet states of $\mathrm{BH}^{+}$and $\mathrm{C}_{2}^{+}$and for the quintet states of $\mathrm{C}_{2}$. The $\mathrm{SAC}-\mathrm{CI}$ general- $R$ method is also extended to the high-spin states, and proved to be useful especially for calculating accurate adiabatic excitation energies of the systems having quasi-degenerate orbital structure. (c) 2001 Published by Elsevier Science B.V.
\end{abstract}

\section{Introduction}

High-spin multiplet states sometimes appear for molecules having degenerate or quasi-degenerate orbital structure. Transition metal complexes and clusters often have high-spin multiplet states in their ground and lower excited states. High-spin multiplet states are also important in the reaction dynamics and energy relaxation processes like predissociation and recombination processes. For investigating molecular geometries, vibrations, chemical reactions, energy relaxation processes, and dynamics in high-spin multiplet states, the information on the derivatives of the adiabatic

\footnotetext{
${ }^{*}$ Corresponding author. Fax: +81-75-753-5910.

E-mail address: hiroshi@subchem.kyoto-u.ac.jp (H. Nakatsuji).
}

potential energy surfaces of molecules in these states are very important. Furthermore, the derivatives of the energy of these states with respect to the external electric and magnetic fields provide electric and magnetic properties of molecules in these electronic states [1]. A purpose of this Letter is to present a reliable method of calculating such energy derivatives by the SAC (symmetry adapted cluster)-CI (configuration-interaction) method for the high-spin multiplet state.

The SAC/SAC-CI method [2-4] was originally published in 1978 for studying ground, excited, ionized and electron-attached (anion) states of molecules and has been successfully applied to various chemistries and physics involving many different kinds of electronic states [5,6]. It has also been applied to multi-electron processes by introducing SAC-CI general- $R$ method [7-10] and to high-spin states from quartet to septet spin mul- 
tiplicities [11,12]. The analytical gradient of the SAC/SAC-CI energy was formulated and implemented in this laboratory at the SD (single double)- $R$ level $[13,14]$, and recently, it has further been extended and implemented by the present authors to include the SAC-CI general- $R$ method [15].

In this study, we develop further the analytical energy gradient of the SAC-CI method to include high-spin multiplet states. As shown in the exponentially generated CI (EGCI) study [12] of the high-spin states, quasi-degenerate orbital structure often appears in the high-spin states of molecules. It is therefore important to extend the SAC-CI general- $R$ method to the high-spin states, even though an ultimate solution would be due to the multi-reference theory [8-10]. The performance of the developed method including the general- $R$ case was examined for the quartet states of $\mathrm{BH}^{+}$and $\mathrm{C}_{2}^{+}$and the quintet states of $\mathrm{C}_{2}$. The details will be given in a forthcoming paper [16].

\section{Theory}

The SAC-CI wavefunction is generated from the correlated SAC ground state as $[3,4,6]$

$\Psi_{\mathrm{SAC}-\mathrm{CI}}^{p}=\sum_{N} d_{N}^{p} R_{N}^{\dagger} \Psi_{\mathrm{SAC}}$,

where $\left\{R_{N}^{\dagger}\right\}$ represents a set of excitation operators and $\left\{d_{N}^{p}\right\}$ their coefficients for the $p$ th excited state. In the present SAC-CI for high-spin multiplicity, the operator $\left\{R_{N}^{\dagger}\right\}$ generates high-spin multiplet state from the closed-shell SAC state. Since this process is described by multi-electron processes [11], the symmetry-adapted linked operators for the quartet to septet spin states are written by the multiple excitation operators summarized in Table 1, which gives only lower operators. Using these operators, the SAC-CI wavefunctions for highspin multiplicities are expressed as

Quartet :

$$
{ }^{4} \Psi_{\mathrm{SAC}-\mathrm{CI}}=\left(\sum_{(i j a)} d_{i j}^{a 4} R_{i j}^{a}+\sum_{(i j k a b)} d_{i j k}^{a b 4} R_{i j k}^{a b}\right) \Psi_{\mathrm{SAC}}
$$

Quintet :

$$
{ }^{5} \Psi_{\mathrm{SAC}-\mathrm{CI}}=\left(\sum_{(i j a b)} d_{i j}^{a b 5} R_{i j}^{a b}+\sum_{(i j k a b c)} d_{i j k}^{a b c 5} R_{i j k}^{a b c}\right) \Psi_{\mathrm{SAC}},
$$

Sextet :

$$
{ }^{6} \Psi_{\mathrm{SAC}-\mathrm{CI}}=\left(\sum_{(i j k a b)} d_{i j k}^{a b 6} R_{i j k}^{a b}+\sum_{(i j k l a b c)} d_{i j k l}^{a b c 6} R_{i j k l}^{a b c}\right) \Psi_{\mathrm{SAC}},
$$

and

Septet :

$$
{ }^{7} \Psi_{\mathrm{SAC}-\mathrm{CI}}=\left(\sum_{(i j k a b c)} d_{i j k}^{a b c 7} R_{i j k}^{a b c}+\sum_{(i j k l a b c d)} d_{i j k l}^{a b c d 7} R_{i j k l}^{a b c d}\right) \Psi_{\mathrm{SAC}}
$$

where the parentheses mean that the summation is limited to the non-redundant linked excitation operators.

The SAC-CI equation is obtained by projecting the Schrödinger equation for the SAC-CI wavefunction onto the space of the linked excited configurations as

$\left\langle 0\left|R_{K}\left(H-E_{\mathrm{SAC}-\mathrm{CI}}^{p}\right)\right| \Psi_{\text {SAC-CI }}^{p}\right\rangle=0$,

Table 1

Lower $R$-operators for the SAC-CI calculation [11]

\begin{tabular}{lll}
\hline Spin multiplicity & Linked operators & \\
\hline Quartet & ${ }^{4} R_{i j}^{a}=a_{a \alpha}^{\dagger} a_{i \beta} a_{j \beta}$ & ${ }^{4} R_{i j k}^{a b}=S_{i}^{a 4} R_{j k}^{b}$ \\
Quintet & ${ }^{5} R_{i j}^{a b}=a_{a \alpha}^{\dagger} a a_{b \alpha}^{\dagger} a_{j \beta} a_{i \beta}$ & ${ }^{5} R_{i j k}^{a b c}=S_{i}^{a 5} R_{j k}^{b c}$ \\
Sextet & ${ }^{6} R_{i j k}^{a b}=a_{a \alpha}^{\dagger} a a_{b \alpha}^{\dagger} a_{j \beta} a_{i \beta} a_{k \beta}$ & ${ }^{6} R_{i j k c}^{a b c}=S_{i}^{a 6} R_{j k k}^{b c}$ \\
Septet & ${ }^{7} R_{i j k}^{a b c}=a_{a \alpha}^{\dagger} a_{b \alpha}^{\dagger} a_{c \alpha}^{\dagger} a_{k \beta} a_{j \beta} a_{i \beta}$ & ${ }^{7} R_{i j k l}^{a b c d}=S_{i}^{a 7} R_{j k l}^{b c d}$ \\
\hline
\end{tabular}

${ }^{\mathrm{a}}$ The operator $S_{i}^{a}$ denotes singlet-type single excitation operator; $S_{i}^{a}=\left(a_{a \alpha}^{\dagger} a_{i \alpha}+a_{a \beta}^{\dagger} a_{i \beta}\right) / \sqrt{2}$. 
where $E_{\mathrm{SAC}-\mathrm{CI}}^{p}$ is the SAC-CI energy of $p$ th excited state. Neglecting some less important unlinked integrals [14], the SAC-CI energy measured from the Hartree-Fock energy of the SAC state, $\Delta E_{\mathrm{SAC}-\mathrm{CI}}^{p}$ is derived from Eq. (1) as

$\Delta E_{\mathrm{SAC}-\mathrm{CI}}^{p}=\sum_{M} \sum_{N} d_{M}^{L(p)} d_{N}^{R(p)}\left(H_{M N}+\sum_{I} C_{I} H_{M, N I}\right)$,

where $\Delta E_{\mathrm{SAC}-\mathrm{CI}}^{p}=E_{\mathrm{SAC}-\mathrm{CI}}^{p}-E_{\mathrm{HF}}, H_{M N}$ and $H_{M, N I}$ are the Hamiltonian matrices defined by $\left\langle 0\left|R_{M} H R_{N}^{\dagger}\right| 0\right\rangle$ and $\left\langle 0\left|R_{M} H R_{N}^{\dagger} S_{I}^{\dagger}\right| 0\right\rangle$, respectively, $d_{M}^{\mathrm{L}}$ and $d_{N}^{\mathrm{R}}$ are the SAC-CI left- and right-vectors, and $C_{I}$ is the SAC coefficient. In this Letter, we adopt the convention that the subscripts $I, J, K, L$ refer to the SAC excitation operators $S^{\dagger}$, while $M, N$ the SAC-CI excitation operators $R^{\dagger}$ summarized in Table 1.

The first derivative of the SAC-CI correlation energy with respect to the external parameter $a$ is given in the Hamiltonian matrix form as [14]:

$$
\begin{aligned}
\frac{\Delta E_{\mathrm{SAC}-\mathrm{CI}}}{\partial a}= & \sum_{I}\left\{\sum_{K} Z_{K}^{\mathrm{SAC}-\mathrm{CI}}\left(\sum_{J} C_{J} S_{K J}\right) C_{I}\right. \\
& \left.-Z_{I}^{\mathrm{SAC}-\mathrm{CI}}\right\} \frac{\partial H_{0 I}}{\partial a}+\sum_{M} \sum_{N} d_{M}^{\mathrm{L}} d_{N}^{\mathrm{R}} \\
& \times \frac{\partial H_{M N}}{\partial a}-\sum_{K} \sum_{I} Z_{K}^{\mathrm{SAC}-\mathrm{CI}} C_{I} \frac{\partial H_{K I}}{\partial a} \\
& +\sum_{M} \sum_{N} \sum_{I} d_{M}^{\mathrm{L}} d_{N}^{\mathrm{R}} C_{I} \frac{\partial H_{M, N I}}{\partial a} \\
& -\frac{1}{2} \sum_{K} \sum_{I} \sum_{J} Z_{K}^{\mathrm{SAC}-\mathrm{CI}} C_{I} C_{J} \frac{\partial H_{K, I J}}{\partial a},
\end{aligned}
$$

where $S_{I J}$ represents the overlap matrix that is independent of the external parameters, and $H_{0 I}, H_{I J}$ and $H_{I, J K}$ are the Hamiltonian matrices defined by $\left\langle 0\left|H S_{I}^{\dagger}\right| 0\right\rangle, \quad\left\langle 0\left|S_{I} H S_{J}^{\dagger}\right| 0\right\rangle \quad$ and $\left\langle 0\left|S_{I} H S_{J}^{\dagger} S_{K}^{\dagger}\right| 0\right\rangle$, respectively. $Z_{K}^{\text {SAC-CI }}$ is a component of the SAC-CI $Z$-vector and is calculated from the following simultaneous linear equation [14]:

$$
\begin{aligned}
\sum_{K} & \left\{H_{K I}-\left(\sum_{J} C_{J} S_{K J}\right) H_{0 I}-\Delta E_{\mathrm{SAC}} S_{K I}\right. \\
& \left.+\sum_{J} C_{J} H_{K, I J}\right\} Z_{K}^{\mathrm{SAC}-\mathrm{CI}} \\
= & \sum_{M} \sum_{N} d_{M}^{\mathrm{L}} d_{N}^{\mathrm{R}} H_{M, N I}
\end{aligned}
$$

In the SAC-CI analytical energy gradient method, explicit calculation of the first derivative of the SAC coefficient $\partial C_{I} / \partial a$ is circumvented by using the interchange technique [17] or the socalled $Z$-vector method [18]. The energy gradient for the high-spin state is evaluated from Eqs. (8) and (9).

The first derivative of the Hamiltonian matrix element is expressed simply in terms of one- and two-electron coupling constants as

$\frac{\partial H_{X Y}}{\partial a}=\sum_{i j}^{M O} \gamma_{i j}^{X Y} \frac{\partial f_{i j}}{\partial a}+\sum_{i j k l}^{M O} \Gamma_{i j k l}^{X Y} \frac{\partial(i j \mid k l)}{\partial a}$,

where the subscripts $i, j, k, l$ refer to the spatial orbitals, and $f_{i j}$ and $(i j \mid k l)$ denote fock matrix element and two-electron $M O$ integral, respectively. The matrix elements $\gamma_{i j}^{X Y}$ and $\Gamma_{i j k l}^{X Y}$ are the one- and two-electron coupling constants between configuration functions $\Phi_{X}$ and $\Phi_{Y}$ and are independent of the parameter $a \cdot \gamma_{i j}^{X Y}$ and $\Gamma_{i j k l}^{X Y}$ for high-spin multiplicities are evaluated using the combined algorithm of the projective reduction formalism for bonded functions developed by Reeves and others [19-21], and our own formalism [22] based on the table-CI type idea [23].

Using Eq. (10), we can now rewrite Eq. (8) in an $M O$ representation in terms of the effective density matrices (EDMs):

$$
\begin{aligned}
\frac{\partial \Delta E_{\mathrm{SAC}-\mathrm{CI}}}{\partial a}= & \sum_{i j}^{M O} \gamma_{i j}^{\mathrm{SAC}-\mathrm{CI}} \frac{\partial f_{i j}}{\partial a} \\
& +\sum_{i j k l}^{M O} \Gamma_{i j k l}^{\mathrm{SAC}-\mathrm{CI}} \frac{\partial(i j \mid k l)}{\partial a} .
\end{aligned}
$$

The matrix elements of the EDMs $\gamma_{i j}^{\text {SAC-CI }}$ and $\Gamma_{i j k l}^{\mathrm{SAC}-\mathrm{CI}}$ are represented as 


$$
\begin{aligned}
\gamma_{i j}^{\mathrm{SAC}-\mathrm{CI}} \equiv & \sum_{I}\left\{\sum_{K} Z_{K}^{\mathrm{SAC}-\mathrm{CI}}\left(\sum_{J} C_{J} S_{K J}\right) C_{I}\right. \\
& \left.-Z_{I}^{\mathrm{SAC}-\mathrm{CI}}\right\} \gamma_{i j}^{0 I}+\sum_{M} \sum_{N} d_{M}^{\mathrm{L}} d_{N}^{\mathrm{R}} \gamma_{i j}^{M N} \\
& -\sum_{K} \sum_{I} Z_{K}^{\mathrm{SAC}-\mathrm{CI}} C_{I} \gamma_{i j}^{K I} \\
& +\sum_{M} \sum_{N} \sum_{I} d_{M}^{\mathrm{L}} d_{N}^{\mathrm{R}} C_{I} \gamma_{i j}^{M, N I} \\
& -\frac{1}{2} \sum_{K} \sum_{I} \sum_{J} Z_{K}^{\mathrm{SAC}-\mathrm{CI}} C_{I} C_{J} \gamma_{i j}^{K, I J},
\end{aligned}
$$

and

$$
\begin{aligned}
\Gamma_{i j k l}^{\mathrm{SAC}-\mathrm{CI}} \equiv & \sum_{I}\left\{\sum_{K} Z_{K}^{\mathrm{SAC}-\mathrm{CI}}\left(\sum_{J} C_{J} S_{K J}\right) C_{I}\right. \\
& \left.-Z_{I}^{\mathrm{SAC}-\mathrm{CI}}\right\} \Gamma_{i j k l}^{0 I} \\
& +\sum_{M} \sum_{N} d_{M}^{\mathrm{L}} d_{N}^{\mathrm{R}} \Gamma_{i j k l}^{M N} \\
& -\sum_{K} \sum_{I} Z_{K}^{\mathrm{SAC}-\mathrm{CI}} C_{I} \Gamma_{i j k l}^{K I} \\
& +\sum_{M} \sum_{N} \sum_{I} d_{M}^{\mathrm{L}} d_{N}^{\mathrm{R}} C_{I} \Gamma_{i j k l}^{M, N I} \\
& -\frac{1}{2} \sum_{K} \sum_{I} \sum_{J} Z_{K}^{\mathrm{SAC}-\mathrm{CI}} C_{I} C_{J} \Gamma_{i j k l}^{K, J J},
\end{aligned}
$$

respectively [13,14], where $M, N$ run over the excitation operators of the SAC-CI high-spin expansions (Eqs. (2)-(5)), and $I, J, K$ over those of the SAC expansion.

The SAC-CI general- $R$ method has also been extended to the present SAC-CI gradient code for high-spin states. For example, $R_{N}^{\dagger}$ operators of quartet and quintet spin states include not only double $\left({ }^{4} R_{i j}^{a}\right.$ and $\left.{ }^{5} R_{i j}^{a b}\right)$ and triples $\left({ }^{4} R_{i j k}^{a b}\right.$ and $\left.{ }^{5} R_{i j k}^{a b c}\right)$ but also quadruple $\left({ }^{4} R_{i j k l}^{a b c}\right.$ and $\left.{ }^{5} R_{i j k l}^{a b c d}\right)$ and higherorder excitation operators. In the general- $R$ calculations, the unlinked terms that are redundant with the linked terms are dropped out.

The high-spin SAC-CI analytical energy gradient code has been implemented in the SAC-CI96 program system [24], which has further been incorporated into the development version of the GAUSSIAN suite of programs [25]. The numerical check was done by confirming that the calculated analytical energy gradient is equal to the numerical differentiation of the energy within computational accuracy.

\section{Applications}

\subsection{Quartet states of $\mathrm{BH}^{+}$}

First, we apply the SAC-CI high-spin analytical energy gradient method to the calculation of the spectroscopic constants of the quartet states of $\mathrm{BH}^{+}$. Though there are no experimental data for these quartet states, they were theoretically studied by the multi-configurational self-consistent field $(\mathrm{MCSCF})+\mathrm{CI}$ method [26] and two of them, $\mathrm{a}^{4} \Pi$ and $b^{4} \Sigma^{-}$states, were found to be bound states. The basis set is the double-zeta plus polarization (DZP) basis of Huzinaga-Dunning [27,28], (9s5p1d/4s1p)/[4s2p1d/2s1p]. All the MOs were included in the active space and no configuration selection was performed. The quartet states of $\mathrm{BH}^{+}$were obtained by the ionizations of the neutral closed-shell state. We used the approximately variational SAC-CI (SAC-CI-V) method.

Table 2 shows the results of the equilibrium internuclear distance $r_{\mathrm{e}}$, the harmonic vibrational frequency $\omega_{\mathrm{e}}$, and the adiabatic excitation energy $T_{\mathrm{e}}\left(\mathrm{b}^{4} \Sigma^{-}-\mathrm{a}^{4} \Pi\right)$, compared with those by the MCSCF-CI method [26]. The values of $\omega_{\mathrm{e}}$ were numerically calculated using the analytical first derivatives. The excitation level denotes the number of electrons involved in the excitation process from the closed-shell ground state and the excitation character is shown by the SAC-CI coefficients of the main configurations. The low-lying $a^{4} \Pi$ state is described by the two-electron process, while the $b^{4} \Sigma^{-}$state is represented by the threeelectron process. Therefore, two types of calculations were performed: DT- $R$ included $R(2)$ and $R(3)$ operators and DTQ- $R$ further included $R(4)$ operators, where the number in the parentheses show the number of excitations in the excitation operator. For the $\mathrm{a}^{4} \Pi$ state, both SAC-CI DT- $R$ and DTQ- $R$ methods gave similar results, which were also the case in the previous application to the low-spin states of one-electron process [15]. On 
Table 2

Excitation levels, equilibrium internuclear distances $\left(r_{\mathrm{e}}\right)$, adiabatic excitation energies $\left(T_{\mathrm{e}}\right)$, and harmonic vibrational frequencies $\left(\omega_{\mathrm{e}}\right)$ for the quartet states of $\mathrm{BH}^{+}$

\begin{tabular}{lllllll}
\hline State & Method & $\begin{array}{l}\text { Excitation } \\
\text { level }\end{array}$ & $\begin{array}{l}r_{\mathrm{e}} \\
(\AA)\end{array}$ & $\begin{array}{l}\omega_{\mathrm{e}} \\
\left(\mathrm{cm}^{-1}\right)\end{array}$ & $\begin{array}{l}T_{\mathrm{e}} \\
(\mathrm{eV})\end{array}$ & Main configuration $^{\mathrm{a}}(|C|>0.3)$ \\
\hline $\mathrm{a}^{4} \Pi$ & SAC-CI DT- $R$ & 2 & 1.735 & 829 & 0.000 & $0.87\left(2 \sigma^{-1} 3 \sigma^{-1} 1 \pi\right)+0.45\left(2 \sigma^{-1} 3 \sigma^{-1} 1 \pi\right)$ \\
& SAC-CI DTQ- $R$ & 2 & 1.743 & 803 & 0.000 & $0.86\left(2 \sigma^{-1} 3 \sigma^{-1} 1 \pi\right)+0.44\left(2 \sigma^{-1} 3 \sigma^{-1} 1 \pi\right)$ \\
& MCSCF-CI & & 1.741 & 765 & 0.000 & \\
$\mathrm{~b}^{4} \Sigma^{-}$ & SAC-CI DT- $R$ & 3 & 1.476 & 1466 & 4.429 & $0.79\left(3 \sigma^{-2} 1 \pi^{2} \sigma^{-1}\right)+0.41\left(3 \sigma^{-2} 2 \sigma^{-1} 1 \pi 2 \pi\right)$ \\
& & & 1.449 & 1575 & 4.132 & $-0.41\left(3 \sigma^{-2} 2 \sigma^{-1} 1 \pi 2 \pi\right)$ \\
& SAC-CI DTQ- $R$ & 3 & & & & $0.78\left(3 \sigma^{-2} 1 \pi^{2} 2 \sigma^{-1}\right)+0.41\left(3 \sigma^{-2} 2 \sigma^{-1} 1 \pi 2 \pi\right)$ \\
& & & 1.451 & 1595 & 4.170 & $-0.41\left(3 \sigma^{-2} 2 \sigma^{-1} 1 \pi 2 \pi\right)$
\end{tabular}

${ }^{\mathrm{a}}$ The electronic configuration and excitation level are given relative to the closed-shell $\mathrm{BH}$, namely, $(\operatorname{core})^{2}(2 \sigma)^{2}(3 \sigma)^{2}$.

${ }^{\mathrm{b}}$ Ref. [26].

the other hand, for the $b^{4} \Sigma^{-}$state, the results of the DT- $R$ method were improved by the DTQ- $R$ method: the effect of including the $R(4)$ operators was $0.03 \AA, 110 \mathrm{~cm}^{-1}$ and $0.3 \mathrm{eV}$ for $r_{\mathrm{e}}, \omega_{\mathrm{e}}$ and $T_{\mathrm{e}}$, respectively. This improvement was not so remarkable in comparison with the low-spin states of multi-electron process [15], since the system is small and the variational space of the DT- $R$ calculation is relatively large. The present results for these two states agree reasonably well with those of the MCSCF-CI method [26].

\subsection{Quartet states of $C_{2}^{+}$and quintet states of $C_{2}$}

Next, we examine the performance of the present method for the quartet states of $\mathrm{C}_{2}^{+}$and the quintet states of $\mathrm{C}_{2}$ using, in this case, the perturbation selection technique. The basis set is the correlation-consistent polarized valence triplezeta set (cc-pVTZ) without $f$ polarization function $[29,30],(10 \mathrm{~s} 5 \mathrm{p} 2 \mathrm{~d}) /[4 \mathrm{~s} 3 \mathrm{p} 2 \mathrm{~d}]$. The SAC-CI-V highspin calculations were performed, including all the $M O s$ in the active space. The perturbation selection was due to the state-selection scheme [31]. For the ground state of $\mathrm{C}_{2}$, the energy threshold for the linked operator was $1 \times 10^{-7}$ a.u. and the unlinked terms were written as the products of the important linked terms whose DTCI coefficients are larger than 0.005 . For the quartet and quintet states, the thresholds for $R(2), R(3)$ and $R(4)$ operators were $1 \times 10^{-7}$ a.u. and the thresholds of the CI coefficients for calculating the unlinked operators in the SAC-CI method were 0.005 and 0.0 for $R$ and $S$ operators, respectively. When the perturbation selection is performed at every geometry, the potential energy surface may become discontinuous. Therefore, during the geometry optimization, we used the common set of $R$ and $S$ excitation operators, which were determined by the perturbation selection for the initial geometry. We used the experimental geometry as the initial one. When experimental geometry is not available, the optimized geometry at the DT-CI/DTQ-CI level is used as the initial one. We have found that the dependence of the results on the initial geometry was negligibly small as far as proper geometries were used. In this calculation, we confirmed the convergence of the optimized geometry: geometry optimization was again performed using the excitation operators determined by the perturbation selection at the optimized geometry.

First, we discuss the quartet states of $\mathrm{C}_{2}^{+}$, whose ground is $\mathrm{X}^{4} \Sigma_{\mathrm{g}}^{+}$[32]. The $\mathrm{X}^{4} \Sigma_{\mathrm{g}}^{+}-\mathrm{B}^{4} \Sigma_{\mathrm{u}}^{-}$ electronic transition was studied by laser excitation spectroscopy and the spectroscopic constants of these states were determined [32]. Theoretically, some of the quartet states of $\mathrm{C}_{2}^{+}$have also been studied by the multi-reference (MR)-CI [33,34] and $\operatorname{CCSD}(\mathrm{T})$ methods [35]. Table 3 summarizes $r_{\mathrm{e}}, \omega_{\mathrm{e}}$ and $T_{\mathrm{e}}$ for the $\mathrm{X}^{4} \Sigma_{\mathrm{g}}^{+}, \mathrm{A}^{4} \Pi_{\mathrm{g}}$ and $\mathrm{B}^{4} \Sigma_{\mathrm{u}}^{-}$states of $\mathrm{C}_{2}^{+}$calculated by the SAC-CI method in comparison with the experimental and other theoretical values. 
Table 3

Excitation levels, equilibrium internuclear distances $\left(r_{\mathrm{e}}\right)$, adiabatic excitation energies $\left(T_{\mathrm{e}}\right)$, and harmonic vibrational frequencies $\left(\omega_{\mathrm{e}}\right)$ for the quartet ground and excited states of $\mathrm{C}_{2}^{+}$

\begin{tabular}{|c|c|c|c|c|c|c|}
\hline State & Method & Excitation level & $\begin{array}{l}r_{\mathrm{e}} \\
(\AA)\end{array}$ & $\begin{array}{l}\omega_{\mathrm{e}} \\
\left(\mathrm{cm}^{-1}\right)\end{array}$ & $\begin{array}{l}T_{\mathrm{e}} \\
(\mathrm{eV})\end{array}$ & Main configuration $^{\mathrm{a}}(|C|>0.3)$ \\
\hline $\mathrm{X}^{4} \Sigma_{\mathrm{g}}^{-}$ & $\begin{array}{l}\text { SAC-CI DT- } R \\
\text { SAC-CI DTQ- } R \\
\text { MRCI }^{\text {b }} \\
\text { MRCI }^{\mathrm{c}} \\
{\text { CCSD }(T)^{\mathrm{d}}} \\
\text { Exptl. }^{\mathrm{e}}\end{array}$ & $\begin{array}{l}2 \\
2\end{array}$ & $\begin{array}{l}1.406 \\
1.407 \\
1.413 \\
1.411 \\
1.402 \\
1.403\end{array}$ & $\begin{array}{l}1344 \\
1383 \\
1360 \\
1335 \\
1369 \\
1351\end{array}$ & $\begin{array}{l}0.000 \\
0.000 \\
0.000 \\
0.000 \\
0.000 \\
0.000\end{array}$ & $\begin{array}{l}0.93\left(1 \pi_{\mathrm{u}}^{-2} 3 \sigma_{\mathrm{g}}\right) \\
0.89\left(1 \pi_{\mathrm{u}}^{-2} 3 \sigma_{\mathrm{g}}\right)\end{array}$ \\
\hline $\mathrm{A}^{4} \Pi_{\mathrm{g}}$ & $\begin{array}{l}\text { SAC-CI DT- } R \\
\text { SAC-CI DTQ- } R \\
\text { MRCI }^{\text {b }} \\
\text { MRCI }^{\mathrm{c}} \\
\operatorname{CCSD}(\mathrm{T})^{\mathrm{d}}\end{array}$ & $\begin{array}{l}2 \\
2\end{array}$ & $\begin{array}{l}1.246 \\
1.250 \\
1.270 \\
1.259 \\
1.254\end{array}$ & $\begin{array}{l}2038 \\
1939 \\
1840 \\
1874 \\
1924\end{array}$ & $\begin{array}{l}0.915 \\
1.243 \\
1.370 \\
1.200 \\
1.164\end{array}$ & $\begin{array}{l}0.93\left(2 \sigma_{\mathrm{u}}^{-1} 1 \pi_{\mathrm{u}}^{-1} 3 \sigma_{\mathrm{g}}\right) \\
0.90\left(2 \sigma_{\mathrm{u}}^{-1} 1 \pi_{\mathrm{u}}^{-1} 3 \sigma_{\mathrm{g}}\right)\end{array}$ \\
\hline $\mathrm{B}^{4} \Sigma_{\mathrm{u}}^{-}$ & $\begin{array}{l}\text { SAC-CI DT- } R \\
\text { SAC-CI DTQ- } R \\
\text { MRCI }^{\mathrm{b}} \\
\text { MRCI }^{\mathrm{c}} \\
\operatorname{CCSD}(\mathrm{T})^{\mathrm{d}} \\
\text { Exptl. }^{\mathrm{e}}\end{array}$ & $\begin{array}{l}3 \\
3\end{array}$ & $\begin{array}{l}1.323 \\
1.349 \\
1.355 \\
1.352 \\
1.343 \\
1.347\end{array}$ & $\begin{array}{l}1659 \\
1539 \\
1470 \\
1507 \\
1553 \\
1508\end{array}$ & $\begin{array}{l}5.125 \\
2.617 \\
2.500 \\
2.470 \\
2.488 \\
2.436\end{array}$ & $\begin{array}{l}0.88\left(2 \sigma_{\mathrm{u}}^{-1} 1 \pi_{\mathrm{u}}^{-2} 3 \sigma_{\mathrm{g}}^{2}\right) \\
0.85\left(2 \sigma_{\mathrm{u}}^{-1} 1 \pi_{\mathrm{u}}^{-2} 3 \sigma_{\mathrm{g}}^{2}\right)\end{array}$ \\
\hline
\end{tabular}

${ }^{\mathrm{a}}$ The electronic configuration and excitation level are given relative to the closed-shell $\mathrm{C}_{2}$, namely, $(\operatorname{core})^{4}\left(2 \sigma_{\mathrm{g}}\right)^{2}\left(2 \sigma_{\mathrm{u}}\right)^{2}\left(1 \pi_{\mathrm{u}}\right)^{4}$.

${ }^{\mathrm{b}}$ Ref. [33].

${ }^{\mathrm{c}}$ Ref. [34].

${ }^{\mathrm{d}}$ Ref. [35].

${ }^{\mathrm{e}}$ Ref. [32].

The excitation level means that the SAC-CI main configurations of $\mathrm{X}^{4} \Sigma_{\mathrm{g}}^{+}$and $\mathrm{A}^{4} \Pi_{\mathrm{g}}$ are produced by the two-electron processes from the closed-shell state, while that of $\mathrm{B}^{4} \Sigma_{\mathrm{u}}^{-}$by the threeelectron process. For the ground state $\mathrm{X}^{4} \Sigma_{\mathrm{g}}^{+}$, both
SAC-CI DT- $R$ and DTQ- $R$ methods gave excellent results in comparison with the experiment: the effect of the quadruples was negligible and the deviations from the experiment were $0.004 \AA$ and $32 \mathrm{~cm}^{-1}$ for $r_{\mathrm{e}}$ and $\omega_{\mathrm{e}}$, respectively. For $\mathrm{A}^{4} \Pi_{\mathrm{g}}$

Table 4

Excitation levels, equilibrium internuclear distances $\left(r_{\mathrm{e}}\right)$, adiabatic excitation energies $\left(T_{\mathrm{e}}\right)$, and harmonic vibrational frequencies $\left(\omega_{\mathrm{e}}\right)$ for the quintet states of $\mathrm{C}_{2}$

\begin{tabular}{lllllll}
\hline State & Method & Excitation level & $\begin{array}{l}r_{\mathrm{e}} \\
(\AA)\end{array}$ & $\begin{array}{l}\omega_{\mathrm{e}} \\
\left(\mathrm{cm}^{-1}\right)\end{array}$ & $\begin{array}{l}T_{\mathrm{e}} \\
(\mathrm{eV})\end{array}$ & Main configuration ${ }^{a}(|C|>0.3)$ \\
\hline $\mathrm{X}^{1} \Sigma_{\mathrm{g}}^{+}$ & SAC & 0 & 1.245 & 1875 & 0.000 & \\
& Exptl. & 0 & 1.243 & 1855 & 0.000 & \\
$1^{5} \Pi_{\mathrm{g}}^{+}$ & SAC-CI DT- $R$ & 2 & 1.580 & 936 & 3.235 & $0.94\left(1 \pi_{\mathrm{u}}^{-2} 3 \sigma_{\mathrm{g}} 1 \pi_{\mathrm{g}}\right)$ \\
& SAC-CI DTQ- $R$ & 2 & 1.572 & 983 & 3.082 & $0.91\left(1 \pi_{\mathrm{u}}^{-2} 3 \sigma_{\mathrm{g}} 1 \pi_{\mathrm{g}}\right)$ \\
$1^{5} \Sigma_{\mathrm{g}}^{+}$ & SAC-CI DT- $R$ & 2 & 1.364 & 1472 & 4.233 & $0.64\left(2 \sigma_{\mathrm{u}}^{-1} 1 \pi_{\mathrm{u}}^{-1} 3 \sigma_{\mathrm{g}} 1 \pi_{\mathrm{g}}\right)+0.64\left(2 \sigma_{\mathrm{u}}^{-1} 1 \pi_{\mathrm{u}}^{-1} 3 \sigma_{\mathrm{g}} 1 \pi_{\mathrm{g}}\right)$ \\
& SAC-CI DTQ- $R$ & 2 & 1.365 & 1473 & 4.526 & $0.64\left(2 \sigma_{\mathrm{u}}^{-1} 1 \pi_{\mathrm{u}}^{-1} 3 \sigma_{\mathrm{g}} 1 \pi_{\mathrm{g}}\right)+0.61\left(2 \sigma_{\mathrm{u}}^{-1} 1 \pi_{\mathrm{u}}^{-1} 3 \sigma_{\mathrm{g}} 1 \pi_{\mathrm{g}}\right)$ \\
& & & 1.347 & 1595 & 5.750 & $0.66\left(2 \sigma_{\mathrm{u}}^{-1} 1 \pi_{\mathrm{u}}^{-1} 3 \sigma_{\mathrm{g}} 1 \pi_{\mathrm{g}}\right)-0.66\left(2 \sigma_{\mathrm{u}}^{-1} 1 \pi_{\mathrm{u}}^{-1} 3 \sigma_{\mathrm{g}} 1 \pi_{\mathrm{g}}\right)$ \\
$1^{5} \Delta_{\mathrm{g}}$ & SAC-CI DT- $R$ & 2 & 1.347 & 1564 & 5.752 & $0.65\left(2 \sigma_{\mathrm{u}}^{-1} 1 \pi_{\mathrm{u}}^{-1} 3 \sigma_{\mathrm{g}} 1 \pi_{\mathrm{g}}\right)-0.65\left(2 \sigma_{\mathrm{u}}^{-1} 1 \pi_{\mathrm{u}}^{-1} 3 \sigma_{\mathrm{g}} 1 \pi_{\mathrm{g}}\right)$ \\
\hline
\end{tabular}

${ }^{\mathrm{a}}$ The electronic configuration is given relative to $(\text { core })^{4}\left(2 \sigma_{\mathrm{g}}\right)^{2}\left(2 \sigma_{\mathrm{u}}\right)^{2}\left(1 \pi_{\mathrm{u}}\right)^{4}$. 
state, no experimental value was found, but the SAC-CI results were close to those of the MRCI and $\operatorname{CCSD}(\mathrm{T})$ methods. For $\mathrm{B}^{4} \Sigma_{\mathrm{u}}^{-}$state, the DTQ$R$ method drastically improved the results by including $R(4)$ operators, especially for $T_{\mathrm{e}}$. The differences between the DTQ- $R$ and experimental results were $0.002 \AA, 0.18 \mathrm{eV}$ and $31 \mathrm{~cm}^{-1}$ for $r_{\mathrm{e}}, T_{\mathrm{e}}$ and $\omega_{\mathrm{e}}$, respectively. The SAC-CI analytical energy gradient method has well reproduced the experimental and other theoretical results, regardless of the excitation level, when the general- $R$ calculation is performed.

Finally, we calculated the spectroscopic constants of the quintet states of $\mathrm{C}_{2}$ by the present method. For the quintet states of $\mathrm{C}_{2}$, three states, $1^{5} \Pi_{\mathrm{g}}, 1^{5} \Sigma_{\mathrm{g}}^{+}$and $1^{5} \Delta_{\mathrm{g}}$ states, were found to be bound states by the previous EGCI and CAS-CI studies on the high-spin states [12]. Unfortunately, these states have not yet been observed spectroscopically. The SAC-CI results were given in Table 4 with the ground state calculated by the SAC method. Though the ground state of $\mathrm{C}_{2}$ has quasi-degenerate character, the SAC method well reproduced the experimental values of $r_{\mathrm{e}}$ and $\omega_{\mathrm{e}}$. Note that the various electronic states of $\mathrm{C}_{2}$ were studied by the general- $R$ method in [7]. Three quintet states were described by two-electron process, and therefore both SAC-CI DT- $R$ and DTQ- $R$ methods gave similar results.

\section{Summary}

In this Letter, we have developed and implemented the SAC-CI analytical energy gradient method for the high-spin multiplet states from quartet to septet spin states. The reliability and usefulness of the present method have been confirmed from the applications to the quartet states of $\mathrm{BH}^{+}$and $\mathrm{C}_{2}^{+}$, and the quintet states of $\mathrm{C}_{2}$ molecules. The general- $R$ method has also been extended to the high-spin states, which is useful for the system having quasi-degenerate orbital structure. We expect that the present method should be a powerful tool for investigating the dynamics and the properties of molecules in the high-spin multiplet states.

\section{Acknowledgements}

This study has partially been supported by the special fund from the Ministry of Education, Science, Culture, and Sports.

\section{References}

[1] Y. Yamaguchi, Y. Osamura, J.D. Goddard, H.F. Shaefer, A New Dimension to Quantum Chemistry: Analytic Derivative Methods in $\mathrm{Ab}$ Initio Molecular Electronic Structure Theory, Oxford University Press, New York, 1994.

[2] H. Nakatsuji, K. Hirao, J. Chem. Phys. 68 (1978) 2053.

[3] H. Nakatsuji, Chem. Phys. Lett. 59 (1978) 362.

[4] H. Nakatsuji, Chem. Phys. Lett. 67 (1979) 329, 334.

[5] H. Nakatsuji, Acta. Chim. Acad. Sci. Hung. 129 (1992) 719.

[6] H. Nakatsuji, in: J. Leszczynski (Ed.), Computational Chemistry - Review of Current Trends, vol. 2, World Scientific, New Jersey, 1997, p. 62.

[7] H. Nakatsuji, Chem. Phys. Lett. 177 (1991) 331.

[8] H. Nakatsuji, J. Chem. Phys. 83 (1985) 731.

[9] H. Nakatsuji, J. Chem. Phys. 83 (1985) 5743.

[10] H. Nakatsuji, J. Chem. Phys. 94 (1991) 6716.

[11] H. Nakatsuji, M. Ehara, J. Chem. Phys. 98 (1993) 7179.

[12] M. Ehara, H. Nakatsuji, J. Chem. Phys. 99 (1993) 1952.

[13] T. Nakajima, H. Nakatsuji, Chem. Phys. Lett. 280 (1997) 79.

[14] T. Nakajima, H. Nakatsuji, Chem. Phys. 242 (1999) 177.

[15] M. Ishida, K. Toyota, M. Ehara, H. Nakatsuji, Chem. Phys. Lett. 347 (2001) 493.

[16] M. Ishida, K. Toyota, M. Ehara, H. Nakatsuji (in preparation)

[17] A. Dalgarno, A.L. Stewart, Proc. R. Soc. London, Ser. A 247 (1968) 245.

[18] N.C. Handy, H.F. Schaefer III, J. Chem. Phys. 81 (1984) 5031.

[19] C.M. Reeves, Commun. ACM 9 (1966) 276.

[20] I.L. Copper, R. Mcweeny, J. Chem. Phys. 45 (1966) 226.

[21] B.T. Sutcliffe, J. Chem. Phys. 45 (1966) 235.

[22] M. Hada, H. Nakatsuji, to be published.

[23] R.J. Buncker, in: Studies in Physical and Theoretical Chemistry, vol. 21, Elsevier, Amsterdam, 1982, p. 17.

[24] H. Nakatsuji, M. Hada, M. Ehara, J. Hasegawa, T. Nakajima, H. Nakai, O. Kitao, K. Toyota, SAC/SAC-CI program system (SAC-CI96) for calculating ground, excited, ionized, and electron-attached states having singlet to septet spin multiplicities, 1996.

[25] M.J. Frisch, et al., Gaussian 99, Revision A.8, Gaussian, Inc., Pittsburgh, PA, 1998.

[26] I. Kusunoki, Chem. Phys. Lett. 105 (1984) 175.

[27] S. Huzinaga, J. Chem. Phys. 42 (1965) 1293.

[28] T.H. Dunning Jr., J. Chem. Phys. 53 (1970) 2823. 
[29] T.H. Dunning Jr., J. Chem. Phys. 90 (1989) 1007.

[30] D.E. Woon, T.H. Dunning Jr., J. Chem. Phys. 98 (1993) 1358.

[31] K. Toyota, H. Nakatusji (submitted).

[32] J.P. Maier, M. Rösslein, J. Chem. Phys. 88 (1988) 4614.
[33] C. Petrongolo, P.J. Bruna, S.D. Peyerimhoff, R. Buenker, J. Chem. Phys. 74 (1981) 4594.

[34] P. Rosmus, H.-J. Werner, E.-A. Reinsch, M. Larsson, J. Electron. Spectrosc. Relat. Phenom. 41 (1986) 289.

[35] J.D. Watts, R.J. Bartlett, J. Chem. Phys. 96 (1992) 6073. 